Research Article

\title{
Some New Postquantum Integral Inequalities
}

\author{
Yu-Ming Chu, ${ }^{1}$ Muhammad Uzair Awan $\left(D,{ }^{2}\right.$ Sadia Talib, ${ }^{2}$ Sabah Iftikhar $\left(D,{ }^{3}\right.$ \\ and Latifa Riahi ${ }^{4}{ }^{4}$ \\ ${ }^{1}$ Department of Mathematics, Huzhou University, Huzhou 313000, China \\ ${ }^{2}$ Department of Mathematics, Government College University, Faisalabad, Pakistan \\ ${ }^{3}$ Faculty of Science, King Mongkut's University of Technology Thonburi (KMUTT), Bangkok 10140, Thailand \\ ${ }^{4}$ Faculty of Sciences of Tunis, University of Tunis El Manar, Tunis, Tunisia
}

Correspondence should be addressed to Muhammad Uzair Awan; awan.uzair@gmail.com

Received 22 July 2020; Accepted 21 October 2020; Published 6 November 2020

Academic Editor: Ghulam Shabbir

Copyright ( $\odot 2020 \mathrm{Yu}$-Ming Chu et al. This is an open access article distributed under the Creative Commons Attribution License, which permits unrestricted use, distribution, and reproduction in any medium, provided the original work is properly cited.

The goal of this paper is to derive a new generalized postquantum integral identity. Using this new identity as an auxiliary result, we derive some new variants of integral inequalities using $(p, q)$-differentiable preinvex functions. We also point out some special cases of the obtained results which show that our results are quite unifying ones.

\section{Introduction and Preliminaries}

Calculus without limits that is the quantum calculus depends upon finite difference. The main theme in the study of quantum calculus is to obtain the $q$-analogues of mathematical objects which can be recaptured by taking $q \longrightarrow 1^{-}$. Since the appearance of Jackson's study [1], the subject of quantum calculus has emerged as a bridge between mathematics and physics. It has numerous applications in various branches of mathematics and physics, such as in ordinary fractional calculus, orthogonal polynomials, basic hypergeometric functions, combinatorics, the calculus of variations, the theory of relativity, optimal control problems, $q$-difference and $q$-integral equations, and $q$-transform analysis. For some more and interesting details, see [2]. Tariboon and Ntouyas [3] introduced the notion of $q$-derivatives and $q$-integrals on finite intervals and developed several new $q$-analogues of classical integral inequalities. This motivated many researchers, and resultantly, several $q$-analogues of classical inequalities have been obtained using the concept of $q$-integral on the finite interval. For example, Alp et al. [4] obtained a correct $q$-analogue of Hermite-Hadamard's inequality. Recently, Awan et al. [5] obtained a new $q$-integral identity and derived new associated bounds. In order to show the significance of the results, authors have also discussed applications of the main results of the paper. Erden et al. [6] obtained some new quantum integral inequalities using the convexity property of the functions. Liu and Zhuang [7] obtained quantum analogues of trapezoid-like inequalities using twice $q$-differentiable convex functions. Noor et al. [8] obtained the $q$-analogue of Hermite-Hadamard's inequality using the preinvexity property of the functions. Sudsutad et al. [9] obtained quantum trapezoid-like inequalities. Zhang et al. [10] obtained a new generalized quantum integral identity and obtained some new interesting $q$-integral inequalities. Another significant development in the field of quantum calculus is the introduction of postquantum calculus. In quantum calculus, we deal with $q$-number with one base $q$; however, postquantum calculus includes $p$ - and $q$-numbers with two independent variables $p$ and $q$. This was first investigated by Chakarabarti and Jagannathan [11]. It is worth to mention here that quantum calculus cannot be obtained directly by substituting $q$ by $q / p$ in $q$-calculus, but $q$-calculus can be recaptured by taking $p=1$ in $(p, q)$-calculus. Recently, Tunc and Gov [12] gave the notion of $(p, q)$-derivatives and $(p, q)$-integrals on finite intervals as follows.

Definition 1 (see [12]). Let $\Psi: I \longrightarrow \mathbb{R}$ be a continuous function, and let $x \in I$ and $0<q<p \leq 1$. Then, the $(p, q)$-derivative on $I$ of function $\Psi$ at $x$ is defined as 
$D_{p, q}^{R} \Psi(x)=\frac{\Psi(p x+(1-p) e))-\Psi(q x+(1-q) e)}{(p-q)(x-e)}, \quad x \neq e$.

Definition 2 (see [12]). Let $\Psi: I \subset \mathbb{R} \longrightarrow \mathbb{R}$ be a continuous function. Then, $(p, q)$-integral on $I$ is defined as

$$
\begin{aligned}
\int_{e}^{x} \Psi(\lambda) d_{p, q}^{R} \lambda= & (p-q)(x-e) \\
& \sum_{n=0}^{\infty} \frac{q^{n}}{p^{n+1}} \Psi\left(\frac{q^{n}}{p^{n+1}} x+\left(1-\frac{q^{n}}{p^{n+1}}\right) e\right),
\end{aligned}
$$

for $x \in I$.

Convexity is one of the most important and significant notions in mathematical analysis. Although it is very simple in nature, it is very powerful. It has many applications in various areas of pure and applied sciences, such as in economics, management sciences, optimization theory, and engineering sciences. Extensive study on the theory of convexity leads to many new extensions and generalizations of classical concepts of convex functions. Note that convex functions depend on convex sets. In the literature, we can see several new diversified forms of the convex sets. This naturally leads us to several new generalizations of convex functions. Preinvex functions have been proved to be a very useful generalization of classical convexity. To present the definition of preinvex functions, we have to first give the definition of invex sets.

Definition 3 (see [13]). A set $\mathscr{X} \in \mathbb{R}$ is said to be invex with respect to $\zeta$ if

$$
x+t \zeta(y, x) \in \mathscr{X}, \quad \forall x, y \in \mathscr{X}, t \in[0,1] .
$$

Note that if we take $\zeta(y, x)=y-x$, then invexity reduces to convexity. Using invex sets as the domain, Weir and Mond [14] introduced the class of preinvex functions. This class is defined as follows.

Definition 4 (see [14]). A function $\Psi: \mathscr{X} \longrightarrow \mathbb{R}$ is said to be preinvex with respect to $\zeta$ if

$$
\begin{array}{r}
\Psi(x+t \zeta(y, x)) \leq(1-t) \Psi(x)+t \Psi(y), \\
\forall x, y \in \mathscr{X}, t \in[0,1] .
\end{array}
$$

For $\zeta(y, x)=y-x$, the class of preinvex functions reduces to the class of convex functions.

\section{A Key Lemma}

In this section, we derive our key result which will be helpful in obtaining following results of the paper.

Lemma 1. Let $\Psi: \mathscr{B}=[a, a+\xi(b, a)] \longrightarrow \mathbb{R}$ be $a$ $(p, q)$-differentiable function on $\mathscr{B}^{\circ}$ with $\xi(b, a)>0$. If ${ }_{a} D_{p, q} \Psi$ is integrable on $\mathscr{B}$ and $(7 / 8) \leq q<1$, then

$$
\begin{gathered}
\frac{1}{8}\left[3 \Psi\left(\frac{3 a+\xi(b, a)}{3}\right)+3 \Psi\left(\frac{3 a+2 \xi(b, a)}{3}\right)+\Psi(a+\xi(b, a))\right] \\
\quad-\frac{1}{p \xi(b, a)} \int_{a}^{a+p \xi(b, a)} \Psi(x)_{a} d_{p, q} x \\
=\xi(b, a) \int_{0}^{1} \Phi(t)_{a} D_{q} \Psi(a+t \xi(b, a)) \Phi(t)_{0} d_{q} t,
\end{gathered}
$$

where

$$
\Phi(t)= \begin{cases}q t-\frac{1}{8}, & t \in\left[0, \frac{1}{3}\right), \\ q t-\frac{1}{2}, & t \in\left[\frac{1}{3}, \frac{2}{3}\right), \\ q t-\frac{7}{8}, & t \in\left[\frac{2}{3}, 1\right] .\end{cases}
$$

Proof. Let

$$
\begin{aligned}
& \mathcal{S}_{1}=\int_{0}^{1 / 3}\left(q t-\frac{1}{8}\right){ }_{a} D_{q} \Psi(a+t \xi(b, a))_{0} d_{p, q} t, \\
& \mathcal{S}_{2}=\int_{1 / 3}^{2 / 3}\left(q t-\frac{1}{2}\right){ }_{a} D_{q} \Psi(a+t \xi(b, a))_{0} d_{p, q} t, \\
& \mathcal{S}_{3}=\int_{2 / 3}^{1}\left(q t-\frac{7}{8}\right){ }_{a} D_{q} \Psi(a+t \xi(b, a))_{0} d_{p, q} t .
\end{aligned}
$$

A direct computation gives 
Journal of Mathematics

3

$$
\begin{aligned}
& \mathcal{S}_{1}=\int_{0}^{1 / 3}\left(q t-\frac{1}{8}\right)_{a} D_{q} \Psi(a+t \xi(b, a))_{0} d_{p, q} t \\
& =\int_{0}^{1 / 3} q t_{a} D_{q} \Psi(a+t \xi(b, a))_{0} d_{p, q} t-\frac{1}{8} \int_{0}^{1 / 3}{ }_{a} D_{q} \Psi(a+t \xi(b, a))_{0} d_{p, q} t \\
& =\int_{0}^{1 / 3} q \frac{\Psi(a+p t \xi(b, a))-\Psi(a+q t \xi(b, a))}{(p-q) \xi(b, a)}{ }_{0} d_{p, q} t-\frac{1}{8} \int_{0}^{1 / 3} \frac{\Psi(a+p t \xi(b, a))-\Psi(a+q t \xi(b, a))}{t(p-q) \xi(b, a)} d_{p, q} t \\
& =\frac{1}{3} \sum_{n=0}^{\infty} \frac{q^{n+1}}{p^{n+1}} \frac{\Psi\left(a+\left(q^{n} / 3 p^{n}\right) \xi(b, a)\right)-\Psi\left(a+\left(q^{n+1} / 3 p^{n+1}\right) \xi(b, a)\right)}{\xi(b, a)} \\
& -\frac{1}{8} \sum_{n=0}^{\infty} \frac{\Psi\left(a+\left(q^{n} / 3 p^{n}\right) \xi(b, a)\right)-\Psi\left(a+\left(q^{n+1} / 3 p^{n+1}\right) \xi(b, a)\right)}{\xi(b, a)} \\
& =\frac{(q / p) \sum_{n=0}^{\infty}\left(q^{n} / p^{n}\right) \Psi\left(a+\left(q^{n} / 3 p^{n}\right) \xi(b, a)\right)-\sum_{n=1}^{\infty}\left(q^{n} / p^{n}\right) \Psi\left(a+\left(q^{n} / 3 p^{n}\right) \xi(b, a)\right)}{3 \xi(b, a)} \\
& -\frac{1}{8} \frac{\sum_{n=0}^{\infty}\left(a+\left(q^{n} / 3 p^{n}\right) \xi(b, a)\right) \Psi-\sum_{n=1}^{\infty} \Psi\left(a+\left(q^{n} / 3 p^{n}\right) \xi(b, a)\right)}{\xi(b, a)} \\
& =\frac{1}{3}\left[\frac{q}{p} \frac{\Psi((3 a+\xi(b, a)) / 3)}{\xi(b, a)}-\frac{p-q}{p} \sum_{n=1}^{\infty} \frac{q^{n}}{p^{n}} \frac{\Psi\left(a+\left(q^{n} / 3 p^{n}\right) \xi(b, a)\right)}{\xi(b, a)}\right]-\frac{1}{8} \cdot \frac{\Psi((3 a+\xi(b, a)) / 3)}{\xi(b, a)} \\
& =\frac{q}{3 p \xi(b, a)} \Psi\left(a+\frac{1}{3} \xi(b, a)\right)-\frac{p-q}{3 p \xi(b, a)} \sum_{n=0}^{+\infty} \frac{q^{n}}{p^{n}} \Psi\left(a+\frac{q^{n}}{3 p^{n}} \xi(b, a)\right)+\frac{p-q}{3 p \xi(b, a)} \Psi\left(a+\frac{1}{3} \xi(b, a)\right) \\
& -\frac{1}{8 \xi(b, a)} \cdot \Psi\left(\frac{3 a+\xi(b, a)}{3}\right)+\frac{p-q}{3 p \xi(b, a)} \Psi\left(a+\frac{1}{3} \xi(b, a)\right)-\frac{1}{8 \xi(b, a)} \cdot \Psi\left(\frac{3 a+\xi(b, a)}{3}\right) \\
& =\frac{5}{24 \xi(b, a)} \Psi\left(a+\frac{1}{3} \xi(b, a)\right)-\frac{p-q}{3 p \xi(b, a)} \sum_{n=0}^{+\infty} \frac{q^{n}}{p^{n}} \Psi\left(a+\frac{q^{n}}{3 p^{n}} \xi(b, a)\right) \\
& =\frac{5}{24 \xi(b, a)} \Psi\left(a+\frac{1}{3} \xi(b, a)\right)-\frac{1}{\xi(b, a)} \int_{0}^{1 / 3} \Psi(a+p t \xi(b, a)) d_{p, q} t \\
& =\frac{5}{24 \xi(b, a)} \Psi\left(a+\frac{1}{3} \xi(b, a)\right)-\frac{1}{p \xi^{2}(b, a)} \int_{a}^{a+p(1 / 3) \xi(b, a)} \Psi(x) d_{p, q} x .
\end{aligned}
$$


On the contrary, one has

$$
\begin{aligned}
\mathcal{S}_{2} & =\int_{1 / 3}^{2 / 3}\left(q t-\frac{1}{2}\right)_{a} D_{q} \Psi(a+t \xi(b, a))_{0} d_{p, q} t \\
& =\int_{0}^{2 / 3}\left(q t-\frac{1}{2}\right)_{a} D_{q} \Psi(a+t \xi(b, a))_{0} d_{p, q} t-\int_{0}^{1 / 3}\left(q t-\frac{1}{2}\right)_{0} d_{p, q} \Psi(a+t \xi(b, a))_{0} d_{p, q} t \\
& =\frac{1}{6} \cdot \frac{\Psi((3 a+\xi(b, a)) / 3)}{\xi(b, a)}+\frac{1}{6 \xi(b, a)} \Psi\left(\frac{3 a+2 \xi(b, a)}{3}\right)-\frac{1}{p \xi^{2}(b, a)} \int_{a+(p / 3) \xi(b, a)}^{a+(2 p / 3) \xi(b, a)} \Psi(x)_{a} d_{q} x, \\
\mathcal{S}_{3} & =\int_{2 / 3}^{1}\left(q t-\frac{7}{8}\right)_{a} D_{q} \Psi(a+t \xi(b, a))_{0} d_{p, q} t \\
& =\int_{0}^{1}\left(q t-\frac{7}{8}\right)_{a} D_{q} \Psi(a+t \xi(b, a))_{0} d_{p, q} t-\int_{0}^{2 / 3}\left(q t-\frac{7}{8}\right)_{a} D_{q} \Psi(a+t \xi(b, a))_{0} d_{p, q} t \\
& =\frac{5}{24} \cdot \frac{\Psi(3 a+2 \xi(b, a) / 3)}{\xi(b, a)}+\frac{1}{8 \xi(b, a)} \Psi(a+\xi(b, a))-\frac{1}{p \xi^{2}(b, a)} \int_{a+(2 p / 3) \xi(b, a)}^{a+p \xi(b, a)} \Psi(x)_{a} d_{q} x .
\end{aligned}
$$

This leads to

$$
\begin{aligned}
\int_{0}^{1} \Phi(t)_{a} D_{q} \Psi(a+t \xi(b, a))_{0} d_{p, q} t= & \frac{1}{8}\left[3 \Psi\left(\frac{3 a+\xi(b, a)}{3}\right)+3 \Psi\left(\frac{3 a+2 \xi(b, a)}{3}\right)+\Psi(a+\xi(b, a))\right] \\
& -\frac{1}{p \xi(b, a)} \int_{a}^{a+p \xi(b, a)} \Psi(x)_{a} d_{p, q} x .
\end{aligned}
$$

Multiplying the above equality with $\xi(b, a)$ leads to the desired identity (5). The proof of Lemma 1 is completed.

\section{Main Results}

We now derive our main results.

$$
\begin{aligned}
& \left|\frac{1}{8}\left[3 \Psi\left(\frac{3 a+\xi(b, a)}{3}\right)+3 \Psi\left(\frac{3 a+2 \xi(b, a)}{3}\right)+\Psi(a+\xi(b, a))\right]-\frac{1}{p \xi(b, a)} \int_{a}^{a+p \xi(b, a)} \Psi(x)_{a} d_{p, q} x\right| \\
& \quad \leq \xi(b, a)\left[\left.L_{1}(p, q)\right|_{a} D_{p, q} \Psi(a)\left|+L_{2}(p, q)\right|_{a} D_{p, q} \Psi(b) \mid\right] .
\end{aligned}
$$

where

$$
\begin{aligned}
L_{1}(p, q):= & \frac{1}{6912 q^{2}(p+q)\left(p^{2}+p q+q^{2}\right)}\left[768 q^{5}+13488 q^{4}+27744 p q^{3}-14256 q^{3}-13056 p^{2} q^{3}-13056 p q^{4}+39264 p^{2} q^{2}\right. \\
& \left.-13824 p^{3} q^{2}-14256 p^{2} q-14256 p q^{2}+14256 p^{3} q-11016 p^{2}-11016 q^{2}-11016 p q+11016 q+11016 p\right] \\
L_{2}(p, q):= & \frac{768 q^{4}+768 p q^{3}-10752 p^{2} q^{2}+11016 p^{2}+11016 p q+11016 q^{2}-11016 p-11016 q}{6912 q^{2}(p+q)\left(p^{2}+p q+q^{2}\right)} .
\end{aligned}
$$


Proof. Using Lemma 1 and the assumption condition that ${ }_{a} D_{p, q} \Psi \mid$ is a preinvex function, we have

$$
\begin{aligned}
& \left|\frac{1}{8}\left[3 \Psi\left(\frac{3 a+\xi(b, a)}{3}\right)+3 \Psi\left(\frac{3 a+2 \xi(b, a)}{3}\right)+\Psi(a+\xi(b, a))\right]-\frac{1}{p \xi(b, a)} \int_{a}^{a+p \xi(b, a)} \Psi(x)_{a} d_{p, q} x\right| \\
& =\xi(b, a) \mid \int_{0}^{1 / 3}\left(q t-\frac{1}{8}\right)_{a} D_{p, q} \Psi(a+t \xi(b, a))_{0} d_{p, q} t+\int_{1 / 3}^{2 / 3}\left(q t-\frac{1}{2}\right)_{a} D_{p, q} \Psi(a+t \xi(b, a))_{a} d_{p, q} t \\
& +\int_{2 / 3}^{1}\left(q t-\frac{7}{8}\right)_{a} D_{p, q} \Psi(a+t \xi(b, a))_{0} d_{p, q} t \mid \leq \xi(b, a) \\
& {\left[\left.\left.\int_{0}^{1 / 3}\left|q t-\frac{1}{8}\right|\right|_{a} D_{p, q} \Psi(a+t \xi(b, a))\right|_{0} d_{p, q} t+\left.\left.\int_{1 / 3}^{2 / 3}\left|q t-\frac{1}{2}\right|\right|_{a} D_{p, q} \Psi(a+t \xi(b, a))\right|_{0} d_{p, q} t+\left.\left.\int_{2 / 3}^{1}\left|q t-\frac{7}{8}\right|\right|_{a} D_{p, q} \Psi(a+t \xi(b, a))\right|_{0} d_{p, q} q^{t}\right]} \\
& \leq \xi(b, a)\left[\int_{0}^{1 / 3}\left|q t-\frac{1}{8}\right|\left(\left.(1-t)\right|_{a} D_{p, q} \Psi(a)|+t|_{a} D_{p, q} \Psi(b) \mid\right)_{0} d_{p, q} t+\int_{1 / 3}^{2 / 3}\left|q t-\frac{1}{2}\right|\left(\left.(1-t)\right|_{a} D_{p, q} \Psi(a)|+t|_{a} D_{p, q} \Psi(b) \mid\right)_{0} d_{p, q} t\right. \\
& \left.+\int_{2 / 3}^{1}\left|q t-\frac{7}{8}\right|\left(\left.(1-t)\right|_{a} D_{p, q} \Psi(a)|+t|_{a} D_{p, q} \Psi(b) \mid\right)_{0} d_{p, q} t\right] \\
& =\xi(b, a)\left(\left|{ }_{a} D_{p, q} \Psi(a)\right| \int_{0}^{1 / 3}(1-t)\left|q t-\frac{1}{8}\right|+\left|{ }_{a} D_{p, q} \Psi(b)\right| \int_{0}^{1 / 3} t\left|q t-\frac{1}{8}\right|{ }_{0} d_{p, q} t\right) \\
& +\xi(b, a)\left(\left|{ }_{a} D_{p, q} \Psi(a)\right| \int_{1 / 3}^{2 / 3}(1-t)\left|q t-\frac{1}{2}\right| d_{p, q} t+\left.\right|_{a} D_{p, q} \Psi(b)\left|\int_{1 / 3}^{2 / 3}(1-t)\right| q t-\frac{1}{2} \mid 0 d_{p, q} t\right) \\
& +\xi(b, a)\left(\left|{ }_{a} D_{p, q} \Psi(a)\right| \int_{2 / 3}^{1}(1-t)\left|q t-\frac{7}{8}\right|{ }^{0} d_{p, q} t+\left|{ }_{a} D_{p, q} \Psi(b)\right| \int_{2 / 3}^{1}(1-t)\left|q t-\frac{7}{8}\right|{ }^{0} d_{p, q} t\right) \\
& =\xi(b, a)\left(\frac { 1 } { 6 9 1 2 q ^ { 2 } ( p + q ) ( p ^ { 2 } + p q + q ^ { 2 } ) } \left[480 q^{5}+192 p^{2} q^{3}-288 p^{3} q^{2}+216 p^{3} q+528 p^{2} q^{2}-216 p^{2} q+192 p q^{4}+272 p q^{3}\right.\right. \\
& \left.-216 p q^{2}+56 q^{4}-216 q^{3}-27 p^{2}-27 p q-27 q^{2}+27 p+27 q\right]\left|{ }_{a} D_{p, q} \Psi(a)\right| \\
& \left.+\left.\frac{160 q^{4}+160 p q^{3}-96 p^{2} q^{2}+27 p^{2}+27 p q+27 q^{2}-27 p-27 q}{6912 q^{2}(p+q)\left(p^{2}+p q+q^{2}\right)}\right|_{a} D_{p, q} \Psi(b) \mid\right) \\
& +\xi(b, a)\left(\frac { 1 } { 1 0 8 q ^ { 2 } ( p + q ) ( p ^ { 2 } + p q + q ^ { 2 } ) } \left[6 q^{5}+48 q^{4}-48 p q^{4}-48 p^{2} q^{3} n+102 p q^{3}-54 q^{3}-54 p^{3} q^{2}+138 p^{2} q^{2}-54 p q^{2}-27 q^{2}+54 p^{3} q\right.\right. \\
& \left.-54 p^{2} q-27 p^{2}-27 p q+27 p+27 q\right]\left|D_{p, q} \Psi(a)\right| \\
& \left.+\left.\frac{6 p q^{3}+6 q^{4}-30 p^{2} q^{2}+27 p^{2}+27 p q+27 q^{2}-27 p-27 q}{108 q^{2}(p+q)\left(p^{2}+p q+q^{2}\right)}\right|_{a} D_{p, q} \Psi(b) \mid\right) \\
& +\xi(b, a)\left(\frac { 1 } { 6 9 1 2 q ^ { 2 } ( p + q ) ( p ^ { 2 } + p q + q ^ { 2 } ) } \left[-96 q^{5}+10360 q^{4}+20944 p q^{3}-10584 q^{3}-10176 p^{2} q^{3}-10176 p q^{4}\right.\right. \\
& \left.+29904 p^{2} q^{2}-10080 p^{3} q^{2}-10584 p^{2} q-10584 p q^{2}+10584 p^{3} q-9261 p^{2}-9261 q^{2}-9261 p q+9261 q+9261 p\right]\left|{ }_{a} D_{p, q} \Psi(a)\right| \\
& \left.224 q^{4}+224 p q^{3}-8736 p^{2} q^{2}+9261 p^{2}+9261 p q+\frac{9261 q^{2}-9261 p-9261 q}{6912 q^{2}(p+q)\left(p^{2}+p q+q^{2}\right)}||_{a} D_{p, q} \Psi(b) \mid\right) \\
& =\xi(b, a)\left[\frac { 1 } { 6 9 1 2 q ^ { 2 } ( p + q ) ( p ^ { 2 } + p q + q ^ { 2 } ) } \left[768 q^{5}+13488 q^{4}+27744 p q^{3}-14256 q^{3}-13056 p^{2} q^{3}-13056 p q^{4}+39264 p^{2} q^{2}-13824 p^{3} q^{2}-14256 p^{2} q n\right.\right. \\
& \left.-14256 p q^{2}+14256 p^{3} q-11016 p^{2}-11016 q^{2} n-11016 p q+11016 q+11016 p\right]\left.\right|_{a} D_{p, q} \Psi(a) \mid n \\
& \left.768 q^{4}+768 p q^{3}-10752 p^{2} q^{2}+11016 p^{2}+\frac{11016 p q+11016 q^{2}-11016 p-11016 q}{6912 q^{2}(p+q)\left(p^{2}+p q+q^{2}\right)}\left|{ }_{a} D_{p, q} \Psi(b)\right|\right] .
\end{aligned}
$$


This completes the proof.

Theorem 2. Let $\Psi: \mathscr{B}=[a, a+\xi(b, a)] \longrightarrow \mathbb{R}$ be a q-differentiable function on $\mathscr{B}^{\circ}$ with $\xi(b, a)>0$. If $\left.\left.\right|_{a} D_{p, q} \Psi\right|^{r}$ is an integrable and preinvex function with $(7 / 8) \leq q<1$ and $s^{-1}+r^{-1}=1$, then

$$
\begin{aligned}
& \left|\frac{1}{8}\left[3 \Psi\left(\frac{3 a+\xi(b, a)}{3}\right)+3 \Psi\left(\frac{3 a+2 \xi(b, a)}{3}\right)+\Psi(a+\xi(b, a))\right]-\frac{1}{p \xi(b, a)} \int_{a}^{a+p \xi(b, a)} \Psi(x)_{a} d_{p, q} x\right| \\
& \quad \leq \xi(b, a)\left[\left(\frac{\left[3^{s+1}+(8 q-3)^{s+1}\right](p-q)}{24^{s+1} q\left(p^{s+1}-q^{s+1}\right)}\right)\left(\frac{\left.\left.(3 q+3 p-1)\right|_{a} D_{p, q} \Psi(a)\right|^{r}+\left|{ }_{a} D_{p, q} \Psi(b)\right|^{r}}{9(p+q)}\right)\right)^{1 / r} \\
& \quad+\left(\frac{\left[(3-2 q)^{s+1}+(6 q-5)^{s+1}\right](p-q)}{6^{s+1} q\left(p^{s+1}-q^{s+1}\right)}\right)^{1 / s}\left(\frac{\left.\left.(q+p-1)\right|_{a} D_{p, q} \Psi(a)\right|^{r}+\left|{ }_{a} D_{p, q} \Psi(b)\right|^{r}}{3(p+q)}\right)^{1 / r} \\
& \left.\quad+\left(\frac{\left[(21-16 q)^{s+1}+(24 q-21)^{s+1}\right](p-q)}{24^{s+1} q\left(p^{s+1}-q^{s+1}\right)}\right)^{1 / s}\left(\frac{\left.\left.(3 q+3 p-5)\right|_{a} D_{p, q} \Psi(a)\right|^{r}+\left.\left.5\right|_{a} D_{p, q} \Psi(b)\right|^{r}}{9(p+q)}\right)^{1 / r}\right] .
\end{aligned}
$$

Proof. Using Lemma 1, Hölder's inequality, and the assumption condition that $\left.\left.\right|_{a} D_{p, q} \Psi\right|^{r}$ is a preinvex function, we have

$$
\begin{aligned}
& \left|\frac{1}{8}\left[3 \Psi\left(\frac{3 a+\xi(b, a)}{3}\right)+3 \Psi\left(\frac{3 a+2 \xi(b, a)}{3}\right)+\Psi(a+\xi(b, a))\right]-\frac{1}{p \xi(b, a)} \int_{a}^{a+p \xi(b, a)} \Psi(x)_{a} d_{p, q} x\right| \\
& =\xi(b, a) \mid \int_{0}^{1 / 3}\left(q t-\frac{1}{8}\right)_{a} D_{p, q} \Psi(a+t \xi(b, a))_{0} d_{p, q} t+\int_{1 / 3}^{2 / 3}\left(q t-\frac{1}{2}\right)_{a} D_{p, q} \Psi(a+t \xi(b, a))_{0} d_{p, q} t \\
& +\int_{(a+t \xi(b, a))}^{1}\left(q t-\frac{7}{8}\right){ }_{a} D_{p, q} \Psi(a+t \xi(b, a))_{0} d_{p, q} t \mid \\
& \leq \xi(b, a)\left[\left(\int_{0}^{1 / 3}\left|q t-\frac{1}{8}\right|_{0}^{s} d_{p, q} t\right)^{1 / s}\left(\int_{0}^{1 / 3}\left|{ }_{a} D_{p, q} \Psi(a+t \xi(b, a))\right|_{0}^{r} d_{p, q} t\right)^{1 / r}\right. \\
& +\left(\int_{1 / 3}^{2 / 3}\left|q t-\frac{1}{2}\right|_{0}^{s} d_{p, q} t\right)^{1 / s}\left(\int_{1 / 3}^{2 / 3}\left|{ }_{a} D_{p, q} \Psi(a+t \xi(b, a))\right|_{0}^{r} d_{p, q} t\right)^{1 / r} \\
& +\left(\int_{2 / 3}^{1}\left|q t-\frac{7}{8}\right|_{0}^{s} d_{p, q} t\right)^{1 / s}\left(\int_{2 / 3}^{1}\left|{ }_{a} D_{p, q} \Psi(a+t \xi(b, a))\right|_{0}^{r} d_{p, q} t\right)^{1 / r} \\
& \leq \xi(b, a)\left[\left(\int_{0}^{1 / 3}\left|q t-\frac{1}{8}\right|_{0}^{s} d_{p, q} t\right)^{1 / s}\left(\left|{ }_{a} D_{p, q} \Psi(a)\right|^{r} \int_{0}^{1 / 3}(1-t)_{0} d_{p, q} t+\left.\left.\right|_{a} D_{p, q} \Psi(b)\right|^{r} \int_{0}^{1 / 3} t_{0} d_{p, q} t\right)^{1 / r}\right. \\
& +\left(\int_{1 / 3}^{2 / 3}\left|q t-\frac{1}{2}\right|_{0}^{s} d_{p, q} t\right)^{1 / s}\left(\left|{ }_{a} D_{p, q} \Psi(a)\right|^{r} \int_{1 / 3}^{2 / 3}(1-t)_{0} d_{p, q} t+\left|{ }_{a} D_{p, q} \Psi(b)\right|^{r} \int_{1 / 3}^{2 / 3} t_{0} d_{p, q} t\right)^{1 / r} \\
& \left.+\left(\int_{2 / 3}^{1}\left|q t-\frac{7}{8}\right|_{0}^{s} d_{p, q} t\right)^{1 / s}\left(\left|{ }_{a} D_{p, q} \Psi(a)\right|^{r} \int_{2 / 3}^{1}(1-t)_{0} d_{p, q} t+\left|{ }_{a} D_{p, q} \Psi(b)\right|^{r} \int_{2 / 3}^{1} t_{0} d_{p, q} t\right)^{1 / r}\right] .
\end{aligned}
$$


Now, it is not difficult to show that

$$
\int_{0}^{1 / 3}\left|q t-\frac{1}{8}\right|_{0}^{s} d_{p, q} t=\frac{\left[3^{s+1}+(8 q-3)^{s+1}\right](p-q)}{24^{s+1} q\left(p^{s+1}-q^{s+1}\right)} .
$$

Similarly,

$$
\begin{aligned}
& \int_{1 / 3}^{2 / 3}\left|q t-\frac{1}{8}\right|_{0}^{s} d_{p, q} t 0=\frac{\left[(3-2 q)^{s+1}+(6 q-5)^{s+1}\right](p-q)}{6^{s+1} q\left(p^{s+1}-q^{s+1}\right)}, \\
& \int_{2 / 3}^{1}\left|q t-\frac{1}{8}\right|_{0}^{s} d_{p, q} t=\frac{\left[(21-16 q)^{s+1}+(24 q-21)^{s+1}\right](p-q)}{24^{s+1} q\left(p^{s+1}-q^{s+1}\right)} .
\end{aligned}
$$

Hence,

$$
\begin{aligned}
&\left|\frac{1}{8}\left[3 \Psi\left(\frac{3 a+\xi(b, a)}{3}\right)+3 \Psi\left(\frac{3 a+2 \xi(b, a)}{3}\right)+\Psi(a+\xi(b, a))\right]-\frac{1}{p \xi(b, a)} \int_{a}^{a+p \xi(b, a)} \Psi(x)_{a} d_{p, q} x\right| \\
& \leq \xi(b, a)\left[\left(\frac{\left[3^{s+1}+(8 q-3)^{s+1}\right](p-q)}{24^{s+1} q\left(p^{s+1}-q^{s+1}\right)}\right)^{1 / s}\left(\frac{\left.\left.(3 q+3 p-1)\right|_{a} D_{p, q} \Psi(a)\right|^{r}+\left.\left.\right|_{a} D_{p, q} \Psi(b)\right|^{r}}{9(p+q)}\right)^{1 / r}\right. \\
&+\left(\frac{\left[(3-2 q)^{s+1}+(6 q-5)^{s+1}\right](p-q)}{6^{s+1} q\left(p^{s+1}-q^{s+1}\right)}\right)^{1 / s}\left(\frac{\left.\left.(q+p-1)\right|_{a} D_{p, q} \Psi(a)\right|^{r}+\left.\left.\right|_{a} D_{p, q} \Psi(b)\right|^{r}}{3(p+q)}\right)^{1 / r} \\
&\left.+\left(\frac{\left[(21-16 q)^{s+1}+(24 q-21)^{s+1}\right](p-q)}{24^{s+1} q\left(p^{s+1}-q^{s+1}\right)}\right)^{1 / s}\left(\frac{\left.\left.(3 q+3 p-5)\right|_{a} D_{p, q} \Psi(a)\right|^{r}+\left.\left.5\right|_{a} D_{p, q} \Psi(b)\right|^{r}}{9(p+q)}\right)^{1 / r}\right] .
\end{aligned}
$$

Thus, we deduced the required inequality (14). This completes the proof of Theorem 2.
Theorem 3. Let $\Psi: \mathscr{B}=[a, a+\xi(b, a)] \longrightarrow \mathbb{R}$ be $a$ $(p, q)$-differentiable function on $\mathscr{B}^{\circ}$ with $\xi(b, a)>0$. If $\left.\left.\right|_{a} D_{p, q} \Psi\right|^{r}$ is an integrable and preinvex function with $7 / 8 \leq q<1$ and $r \geq 1$, then

$$
\begin{aligned}
\mid \frac{1}{8}[ & \left.3 \Psi\left(\frac{3 a+\xi(b, a)}{3}\right)+3 \Psi\left(\frac{3 a+2 \xi(b, a)}{3}\right)+\Psi(a+\xi(b, a))\right]-\frac{1}{p \xi(b, a)} \int_{a}^{a+p \xi(b, a)} \Psi(x){ }_{a} d_{p, q} x \mid \\
\leq & \xi(b, a)\left(\frac{20 q^{2}-12 p q+9 p+9 q-9}{288 q(p+q)}\right)^{1-(1 / r)}\left(\left.\left.L_{3(p, q)}\right|_{a} D_{p, q} \Psi(a)\right|^{r}+\left.\left.L_{4}(p, q)\right|_{a} D_{p, q}(b)\right|^{r}\right)^{1 / r} \\
& +\xi(b, a)\left(\frac{q^{2}-9 p q+9 p+9 q-9}{18 q(p+q)}\right)^{1-(1 / r)}\left(\left.\left.L_{5}(p, q)\right|_{a} D_{p, q} \Psi(a)\right|^{r}+\left.\left.L_{6}(p, q)\right|_{a} D_{p, q} \Psi(b)\right|^{r}\right)^{1 / r} \\
& +\xi(b, a)\left(\frac{441 p+441 q-441-420 p q-4 q^{2}}{288 q(p+q)}\right)^{1-(1 / r)}\left(\left.\left.L_{7}(p, q)\right|_{a} D_{p, q} \Psi(a)\right|^{r}+\left.\left.L_{8}(p, q)\right|_{a} D_{p, q} \Psi(b)\right|^{r}\right)^{1 / r},
\end{aligned}
$$


where

$$
\begin{aligned}
& L_{3}(p, q):=\frac{1}{6912 q^{2}(p+q)\left(p^{2}+p q+q^{2}\right)}\left[480 q^{5}+192 p^{2} q^{3}-288 p^{3} q^{2}+216 p^{3} q+528 p^{2} q^{2}-216 p^{2} q+192 p q^{4}\right. \\
& \left.+272 p q^{3}-216 p q^{2}+56 q^{4}-216 q^{3}-27 p^{2}-27 p q-27 q^{2}+27 p+27 q\right] \\
& L_{4}(p, q):=\frac{160 q^{4}+160 p q^{3}-96 p^{2} q^{2}+27 p^{2}+27 p q+27 q^{2}-27 p-27 q}{6912 q^{2}(p+q)\left(p^{2}+p q+q^{2}\right)} \\
& L_{5}(p, q):=\frac{1}{108 q^{2}(p+q)\left(p^{2}+p q+q^{2}\right)}\left[6 q^{5}+48 q^{4}-48 p q^{4}-48 p^{2} q^{3}+102 p q^{3}-54 q^{3}-54 p^{3} q^{2}\right. \\
& \left.+138 p^{2} q^{2}-54 p q^{2}-27 q^{2}+54 p^{3} q-54 p^{2} q-27 p^{2}-27 p q+27 p+27 q\right] \\
& L_{6}(p, q):=\frac{6 p q^{3}+6 q^{4}-30 p^{2} q^{2}+27 p^{2}+27 p q+27 q^{2}-27 p-27 q}{108 q^{2}(p+q)\left(p^{2}+p q+q^{2}\right)} \\
& L_{7}(p, q):=\frac{1}{6912 q^{2}(p+q)\left(p^{2}+p q+q^{2}\right)}\left[-96 q^{5}+10360 q^{4}+20944 p q^{3}-10584 q^{3}-10176 p^{2} q^{3}-10176 p q^{4}+29904 p^{2} q^{2}\right. \\
& \left.-10080 p^{3} q^{2}-10584 p^{2} q-10584 p q^{2}+10584 p^{3} q-9261 p^{2}-9261 q^{2}-9261 p q+9261 q+9261 p\right] \\
& L_{8}(p, q):=\frac{224 q^{4}+224 p q^{3}-8736 p^{2} q^{2}+9261 p^{2}+9261 p q+9261 q^{2}-9261 p-9261 q}{6912 q^{2}(p+q)\left(p^{2}+p q+q^{2}\right)} .
\end{aligned}
$$

Proof. Using Lemma 1, power-mean integral inequality, and the assumption condition that $\left.\left.\right|_{a} D_{p, q} \Psi\right|^{r}$ is a preinvex function, we have

$$
\begin{aligned}
\left|\frac{1}{8}\left[3 \Psi\left(\frac{3 a+\xi(b, a)}{3}\right)+3 \Psi\left(\frac{3 a+2 \xi(b, a)}{3}\right)+\Psi(a+\xi(b, a))\right]-\frac{1}{p \xi(b, a)} \int_{a}^{a+p \xi(b, a)} \Psi(x)_{a} d_{p, q} x\right| \\
=\xi(b, a) \mid \int_{0}^{1 / 3}\left(q t-\frac{1}{8}\right)_{a} D_{p, q} \Psi(a+t \xi(b, a))_{0} d_{p, q} t \\
\quad+\int_{1 / 3}^{2 / 3}\left(q t-\frac{1}{2}\right)_{a} D_{p, q} \Psi(a+t \xi(b, a))_{0} d_{p, q} t+\int_{2 / 3}^{1}\left(q t-\frac{7}{8}\right)_{a} D_{p, q} \Psi(a+t \xi(b, a))_{0} d_{p, q} t \mid \\
\leq \xi(b, a)\left[\left.\left.\int_{0}^{1 / 3}\left|q t-\frac{1}{8}\right|\right|_{a} D_{p, q} \Psi(a+t \xi(b, a))\right|_{0} d_{p, q} t+\left.\left.\int_{1 / 3}^{2 / 3}\left|q t-\frac{1}{2}\right|\right|_{a} D_{p, q} \Psi(a+t \xi(b, a))\right|_{0} d_{p, q} t\right. \\
\left.\quad+\left.\left.\int_{2 / 3}^{1}\left|q t-\frac{7}{8}\right|\right|_{a} D_{p, q} \Psi(a+t \xi(b, a))\right|_{0} d_{p, q} t\right]
\end{aligned}
$$




$$
\begin{aligned}
& \leq \xi A(b, a)\left[\left(\frac{20 q^{2}-12 p q+9 p+9 q-9}{288 q(p+q)}\right)^{1-(1 / r)} \times\left(\left|{ }_{a} D_{p, q} \Psi(a)\right|^{r} \int_{0}^{1 / 3}(1-t)\left|q t-\frac{1}{8}\right|_{0} d_{p, q} t+\left.\left.\right|_{a} D_{p, q} \Psi(b)\right|^{r} \int_{0}^{1 / 3} t\left|q t-\frac{1}{8}\right|{ }^{a} d_{p, q} t\right)^{1 / r}\right. \\
& +\left(\frac{q^{2}-9 p q+9 p+9 q-9}{18 q(p+q)}\right)^{1-(1 / r)} \times\left(\left|{ }_{a} D_{p, q} \Psi(a)\right|^{r} \int_{1 / 3}^{2 / 3}(1-t)\left|q t-\frac{1}{2}\right|{ }_{0} d_{p, q} t+\left|{ }_{a} D_{p, q} \Psi(b)\right|^{r} \int_{1 / 3}^{2 / 3} t\left|q t-\frac{1}{2}\right|{ }_{0} d_{p, q} t\right)^{1 / r} \\
& +\left(\frac{441 p+441 q-441-420 p q-4 q^{2}}{288 q(p+q)}\right)^{1-(1 / r)} \\
& \left.\times\left(\left|{ }_{a} D_{p, q} \Psi(a)\right|^{r} \int_{2 / 3}^{1}(1-t)\left|q t-\frac{7}{8}\right|{ }_{0} d_{p, q} t+\left|{ }_{a} D_{p, q} \Psi(b)\right|^{r} \int_{2 / 3}^{1} t\left|q t-\frac{7}{8}\right|{ }_{0} d_{p, q} t\right)^{1 / r}\right] \\
& =\xi(b, a)\left(\frac{20 q^{2}-12 p q+9 p+9 q-9}{288 q(p+q)}\right)^{1-(1 / r)} \times\left(\frac{1}{6912 q^{2}(p+q)\left(p^{2}+p q+q^{2}\right)}\right. \\
& {\left[480 q^{5}+192 p^{2} q^{3}-288 p^{3} q^{2}+216 p^{3} q+528 p^{2} q^{2}-216 p^{2} q+192 p q^{4}+272 p q^{3}-216 p q^{2}+56 q^{4}-216 q^{3}-27 p^{2}\right.} \\
& \left.\left.-27 p q-27 q^{2}+27 p+27 q\right]\left|D_{a, q} \Psi(a)\right|^{r}+\left.\left.\frac{160 q^{4}+160 p q^{3}-96 p^{2} q^{2}+27 p^{2}+27 p q+27 q^{2}-27 p-27 q}{6912 q^{2}(p+q)\left(p^{2}+p q+q^{2}\right)}\right|_{a} D_{p, q} \Psi(b)\right|^{r}\right)^{1 / r} \\
& \xi(b, a)\left(\frac{q^{2}-9 p q+9 p+9 q-9}{18 q(p+q)}\right)^{1-(1 / r)} \times\left(\frac{1}{108 q^{2}(p+q)\left(p^{2}+p q+q^{2}\right)}\right. \\
& {\left[6 q^{5}+48 q^{4}-48 p q^{4}-48 p^{2} q^{3}+102 p q^{3}-54 q^{3}-54 p^{3} q^{2}+138 p^{2} q^{2}-54 p q^{2}-27 q^{2}+54 p^{3} q-54 p^{2} q\right.} \\
& \left.\left.-27 p^{2}-27 p q+27 p+27 q\right]\left.\left.\right|_{a} D_{p, q} \Psi(a)\right|^{r}+\left.\left.\frac{6 p q^{3}+6 q^{4}-30 p^{2} q^{2}+27 p^{2}+27 p q+27 q^{2}-27 p-27 q}{108 q^{2}(p+q)\left(p^{2}+p q+q^{2}\right)}\right|_{a} D_{p, q} \Psi(b)\right|^{r}\right)^{1 / r} \\
& +\xi(b, a)\left(\frac{441 p+441 q-441-420 p q-4 q^{2}}{288 q(p+q)}\right)^{1-(1 / r)} \times\left(\frac{1}{6912 q^{2}(p+q)\left(p^{2}+p q+q^{2}\right)}\right. \\
& {\left[-96 q^{5}+10360 q^{4}+20944 p q^{3}-10584 q^{3}-10176 p^{2} q^{3}-10176 p q^{4}+29904 p^{2} q^{2}-10080 p^{3} q^{2}-10584 p^{2} q-10584 p q^{2}\right.} \\
& \left.+10584 p^{3} q-9261 p^{2}-9261 q^{2}-9261 p q+9261 q+9261 p\right]\left|{ }_{a} D_{p, q} \Psi(a)\right|^{r} \\
& \left.+\left.\left.\frac{224 q^{4}+224 p q^{3}-8736 p^{2} q^{2}+9261 p^{2}+9261 p q+9261 q^{2}-9261 p-9261 q}{6912 q^{2}(p+q)\left(p^{2}+p q+q^{2}\right)}\right|_{a} D_{p, q} \Psi(b)\right|^{r}\right)^{1 / r} .
\end{aligned}
$$

Thus, we deduced the required inequality (19). This completes the proof of Theorem 3 .

\section{Data Availability}

No data were used to support this study.

\section{Conflicts of Interest}

The authors declare that they have no conflicts of interest.

\section{Authors' Contributions}

All authors contributed equally to the writing of this paper. All authors read and approved the final manuscript.

\section{Acknowledgments}

This work was supported by the Natural Science Foundation of China (Grant nos. 61673169, 11301127, 11701176, 11626101, and 11601485) and the Natural Science Foundation of Huzhou City (Grant no. 2018YZ07).

\section{References}

[1] F. H. Jackson, "On a q-definite integrals," The Quarterly Journal of Pure and Applied Mathematics, vol. 41, pp. 193-203, 1910.

[2] V. Kac, P. Cheung, Quantum Calculus, Springer-Verlag, New York, NY, USA, 2002.

[3] J. Tariboon and S. K. Ntouyas, "Quantum integral inequalities on finite intervals," Journal of Inequalities and Applications, vol. 2014, Article ID 121, 13 pages, 2014. 
[4] N. Alp, M. Z. Sarıkaya, M. Kunt, and İ. İșcan, "q-Hermite Hadamard inequalities and quantum estimates for midpoint type inequalities via convex and quasi-convex functions," Journal of King Saud University - Science, vol. 30, no. 2, pp. 193-203, 2018.

[5] M. U. Awan, S. Talib, A. Kashuri, M. A. Noor, and Y.-M. Chu, "Estimates of quantum bounds pertaining to new $q$-integral identity with applications," Advances in Difference Equations, vol. 2020, p. 424, 2020.

[6] S. Erden, S. Iftikhar, M. R. Delavar, P. Kumam, P. Thounthong, and W. Kumam, "On generalizations of some inequalities for convex functions via quantum integrals," RACSAM, vol. 114, p. 110, 2020.

[7] W.-J. Liu and H.-F. Zhuang, "Some quantum estimates of Hermite-Hadamard inequalities for convex functions," Journal of Applied Analysis and Computation, vol. 7, no. 2, pp. 501-522, 2017.

[8] M. A. Noor, K. I. Noor, and M. U. Awan, "Some quantum integral inequalities via preinvex functions," Applied Mathematics and Computation, vol. 269, pp. 242-251, 2015.

[9] W. Sudsutad, S. K. Ntouyas, and J. Tariboon, "Quantum integral inequalities for convex functions," Journal of Mathematical Inequalities, vol. 9, no. 3, pp. 781-793, 2015.

[10] Y. Zhang, T.-S. Du, H. Wang, and Y.-J. Shen, "Different types of quantum integral inequalities via $(\alpha, m)$-convexity," Journal of Inequalities and Applications, vol. 2018, p. 24, Article ID 264, 2018.

[11] R. Chakrabarti and R. Jagannathan, "A $(p, q)$-oscillator realization of two-parameter quantum algebras," Journal of Physics A: Mathematical and General, vol. 24, no. 13, p. L711, 1991.

[12] M. Tunç and E. Göv, "Some integral inequalities via $(p, q)$-calculus on finite intervals," RGMIA Res. Rep. Coll, vol. 19, p. 12, 2016, https://rgmia.org/papers/v19/v19a95.pdf, Article ID 95.

[13] A. B. Israel and B. Mond, "What is invexity?" Journal of the Australian Mathematical Society, vol. 28B, no. 1, pp. 1-9, 1986.

[14] T. Weir and B. Mond, "Pre-invex functions in multiple objective optimization," Journal of Mathematical Analysis and Applications, vol. 136, no. 1, pp. 29-38, 1988. 